\title{
Importance of alveolar recruitment strategy revisited
}

\author{
Jin Kyoung Kim
}

Department of Anesthesiology and Pain Medicine, Samsung Medical Center, Sungkyunkwan University School of Medicine, Seoul, Korea

Low tidal volume, rather than high tidal volume, ventilation is often used for mechanical ventilation during surgery to avoid lung injury due to alveolar overdistention. Under general anesthesia with a muscle relaxant, there is a risk of compressing the lungs by diaphragmatic displacement, which can be aggravated in patients placed in the supine position. Such a risk becomes even higher during reduced tidal volume ventilation, causing alveolar atelectasis and hypoxemia. Therefore, positive end expiratory pressure (PEEP) is applied to prevent spontaneous closure of alveoli.

In this volume, Jung et al. [1] reports on the importance of the alveolar recruitment strategy (ARS). The authors applied ARS before the start of one-lung ventilation (OLV), and compared the oxygenation score between high- and low-tidal volume groups. Repetitive open and closure of collapsed alveoli by positive pressure ventilation could cause damage to the capillary endothelium and alveolar epithelium. Excessive expansion and risk of alveolar injury may even occur during low tidal volume ventilation due to an uneven distribution of ventilated volume to already open alveoli. However, Jung et al. [1] showed that preemptive ARS commonly increases $\mathrm{PaO}_{2}$ without causing hemodynamic instability in groups with or without PEEP during OLV. The improvement in oxygenation was better in the group where ARS was combined with PEEP. Their study design and intriguing results will draw the attention of general readers of the journal.

The ARS helps to open collapsed alveoli, which is effectively maintained by PEEP. Therefore, both ARS and PEEP maneuvers are compulsory to improve oxygenation and lung mechanics during general anesthesia [2]. Diverse ARS methods have been reported in recent clinical studies. The recommended ARS is peak inspiratory pressure up to $40 \mathrm{cmH}_{2} \mathrm{O}$ at 10 respiratory cycles with 15-20 $\mathrm{cmH}_{2} \mathrm{O}$ PEEP. Adequate PEEP level following ARS is reportedly 5-20 $\mathrm{cmH}_{2} \mathrm{O}$ depending on the condition of the patient (i.e., pulmonary function and physical condition) and also the type of operation.

The time to apply ARS is typically (1) immediately after intubation before confirming lung sounds, (2) after suctioning, or (3) just before extubation. Functional residual capacity is reduced in $50 \%$ of patients after tracheal suctioning, in which case combined ARS and PEEP effectively restores $\mathrm{PaO}_{2}$ [3]. Along with increased $\mathrm{PaO}_{2}$, several reports have indicated that ARS with PEEP during OLV decreases AaDO2, and decreases the alveolar dead space ventilation ratio [4-6]. In addition to OLV anesthesia, ARS with appropriate PEEP is required to improve $\mathrm{PaO}_{2}$ in patients with minimal lung injury during other surgeries with a high risk of atelectasis such as laparoscopic surgery [7-9], pediatric patients [10], and obese patients [9]. This preemptive ARS effect during low tidal volume OLV has been described previously [11]. Preemptive ARS can also be applied before pneumoperitoneum during laparoscopic surgery [8] with the purpose of improving oxygenation.

However, high intrathoracic pressure during ARS may cause hemodynamic instability. Therefore, adjustment of the pressure and application duration should consider a patient's hemodynamic status and the potential risks [5]. In obese patients, higher pressures are required for adequate ARS and PEEP [9]. The risk of hemodynamic derangement by such high pressures can be

Corresponding author: Jin Kyoung Kim, M.D., Ph.D., Department of Anesthesiology and Pain Medicine, Samsung Medical Center, Sungkyunkwan University School of Medicine, 81, Irwon-ro, Gangnam-gu, Seoul 135-710, Korea. Tel: 82-2-3410-2477, Fax: 82-2-3410-6626, E-mail: jkane.kim@samsung.com

(ㄷ) This is an open-access article distributed under the terms of the Creative Commons Attribution Non-Commercial License (http:// creativecommons.org/licenses/by-nc/3.0/), which permits unrestricted non-commercial use, distribution, and reproduction in any medium, provided the original work is properly cited. 
prevented by intravascular volume replacement [12]. Interestingly, one study reported that applying ARS with PEEP to the donor lung in situ as well as after transplantation lowers the risk of primary graft dysfunction [13]. Several reports have suggested the beneficial effects of ARS with PEEP during general abdominal surgery to reduce post-operational complications [14].
In conclusion, applying ARS and PEEP during surgery or in patients vulnerable to atelectasis is helpful for improving oxygenation and lowering postoperative pulmonary complications. PEEP must be sustained to keep alveoli in their open state and optimize patient oxygenation. In addition, adequate ARS before applying PEEP is essential to maximize the benefits of PEEP.

\section{References}

1. Jung JD, Kim SH, Yu BS, Kim HJ. Effects of a preemptive alveolar recruitment strategy on arterial oxygenation during one-lung ventilation with different tidal volumes in patients with normal pulmonary function test. Korean J Anesthesiol 2014; 67: 96-102.

2. Tusman G, Böhm SH, Suarez-Sipmann F, Turchetto E. Alveolar recruitment improves ventilatory efficiency of the lungs during anesthesia. Can J Anaesth 2004; 51: 723-7.

3. Heinze H, Eichler W, Karsten J, Sedemund-Adib B, Heringlake M, Meier T. Functional residual capacity-guided alveolar recruitment strategy after endotracheal suctioning in cardiac surgery patients. Crit Care Med 2011; 39: 1042-9.

4. Unzueta C, Tusman G, Suarez-Sipmann F, Böhm S, Moral V. Alveolar recruitment improves ventilation during thoracic surgery: a randomized controlled trial. Br J Anaesth 2012; 108: 517-24.

5. Cinnella G, Grasso S, Natale C, Sollitto F, Cacciapaglia M, Angiolillo M, et al. Physiological effects of a lung-recruiting strategy applied during one-lung ventilation. Acta Anaesthesiol Scand 2008; 52: 766-75.

6. Tusman G, Böhm SH, Sipmann FS, Maisch S. Lung recruitment improves the efficiency of ventilation and gas exchange during one-lung ventilation anesthesia. Anesth Analg 2004; 98: 1604-9.

7. Cinnella G, Grasso S, Spadaro S, Rauseo M, Mirabella L, Salatto P, et al. Effects of recruitment maneuver and positive end-expiratory pressure on respiratory mechanics and transpulmonary pressure during laparoscopic surgery. Anesthesiology 2013; 118: 114-22.

8. Park HP, Hwang JW, Kim YB, Jeon YT, Park SH, Yun MJ, et al. Effect of pre-emptive alveolar recruitment strategy before pneumoperitoneum on arterial oxygenation during laparoscopic hysterectomy. Anaesth Intensive Care 2009; 37: 593-7.

9. Whalen FX, Gajic O, Thompson GB, Kendrick ML, Que FL, Williams BA, et al. The effects of the alveolar recruitment maneuver and positive end-expiratory pressure on arterial oxygenation during laparoscopic bariatric surgery. Anesth Analg 2006; 102: 298-305.

10. Tusman G, Böhm SH, Tempra A, Melkun F, García E, Turchetto E, et al. Effects of recruitment maneuver on atelectasis in anesthetized children. Anesthesiology 2003; 98: 14-22.

11. Park SH, Jeon YT, Hwang JW, Do SH, Kim JH, Park HP. A preemptive alveolar recruitment strategy before one-lung ventilation improves arterial oxygenation in patients undergoing thoracic surgery: a prospective randomised study. Eur J Anaesthesiol 2011; 28: 298-302.

12. Bohm SH, Thamm OC, von Sandersleben A, Bangert K, Langwieler TE, Tusman G, et al. Alveolar recruitment strategy and high positive end-expiratory pressure levels do not affect hemodynamics in morbidly obese intravascular volume-loaded patients. Anesth Analg 2009; 109: 160-3.

13. Verbeek GL, Myles PS. Intraoperative protective ventilation strategies in lung transplantation. Transplant Rev (Orlando) 2013; $27: 30-5$.

14. Serpa Neto A, Cardoso SO, Manetta JA, Pereira VG, Espósito DC, Pasqualucci Mde O, et al. Association between use of lung-protective ventilation with lower tidal volumes and clinical outcomes among patients without acute respiratory distress syndrome: a meta-analysis. JAMA 2012; 308: 1651-9. 